\title{
On the Magnus Effect
}

\author{
Kern E. Kenyon \\ 4632 North Lane, Del Mar, USA \\ Email: kernken@aol.com
}

Received 11 January 2016; accepted 2 February 2016; published 5 February 2016

Copyright (C) 2016 by author and Scientific Research Publishing Inc.

This work is licensed under the Creative Commons Attribution International License (CC BY).

http://creativecommons.org/licenses/by/4.0/

(c) (i) Open Access

\begin{abstract}
A formula for the Magnus force on a rotating and translating solid cylinder in a fluid is constructed for two different fluid models. In both cases the flow is steady and frictionless with no formation or shedding of eddies behind the cylinder. However, model one is founded on the assumption of irrotationality whereas model two is not but rather makes explicit use of the centrifugal force acting on the curving streamlines above the cylinder. Model two's Magnus force comes out to be 15\% larger in magnitude which is probably more than that can be accounted for by approximations made within the models. Observations will be needed to help decide which model comes closer to the truth. In the force formula the following factors are multiplied together: constant, fluid density, translation speed, and rotation frequency. For model one constant = 2; for model two constant = 2.3.
\end{abstract}

\section{Keywords}

Magnus Effect

\section{Introduction}

Magnus effect, the sideways force on a translating and rotating sphere or cylinder, is discussed in several fluid dynamics textbooks [1]-[3], but confusion can occur when it is combined with other effects, such as friction and eddy forming or shedding. It is within a mixture of different effects that the concept of an "inverse” Magnus effect was introduced [4], which may or may not help with understanding what is going on with some of these fluid flows. Also of the three dictionaries of physics in my possession, only one [5] gives a definition, but that description is so vague that neither the magnitude nor the direction of the Magnus force can be calculated from it. If the OED (Oxford English Dictionary) is consulted [6], under Magnus effect is found: "The effect of rapid spinning on a cylinder moving through a fluid..." Why is the word "rapid” used? One would think that during either rapid spinning or rapid translation or both the extraneous effects of friction and eddy shedding would be relatively more important. In summary, neither have I found anywhere a formula for the "pure” (unadulterated) Magnus force, nor a comparison between theory and measurement. 
These possible deficiencies in the existing literature are addressed in the following essay partly by adapting rotation to a new formulation of the flow past a cylinder that is not founded on the usual assumption of irrotationality. What the newness of the model consists of is the explicit use of the centrifugal force [7]. As a consequence the pressure and velocity fields surrounding the cylinder can both be specified (classically the fluid pressure distribution is usually not mentioned). Thus the sideways pressure force on the cylinder can be computed algebraically. Basic assumptions are: steady inviscid flow with no eddies forming on the back side of the cylinder, and the acceleration of gravity has no bearing on the problem.

\section{Irrotational Magnus Force}

First, a formula for the Magnus force can be constructed from information already available. Consider uniform frictionless horizontal flow of magnitude $U$ moving from left to right past a cylinder of radius $R_{0}$. The cylinder is not rotating on its axis. At the top and bottom of the cylinder the pressure $p$ from Bernoulli's equation is

$$
p=\text { const }-\frac{1}{2} \rho(2 U)^{2}
$$

In (1) the maximum horizontal speed $2 U$ comes from the irrotational solution for flow past a cylinder [8]. The acceleration of gravity does not appear in (1); it has no bearing on what follows.

Next, let the cylinder rotate counter-clockwise with constant angular frequency $\omega$. At the top of the cylinder the pressure $p_{T}$ (relative to the solid surface) is

$$
p_{T}=\text { const }-\frac{1}{2} \rho\left(2 U+\omega R_{0}\right)^{2}
$$

At the bottom of the cylinder the pressure $p_{B}$ is

$$
p_{B}=\text { const }-\frac{1}{2} \rho\left(2 U-\omega R_{0}\right)^{2}
$$

Assume for simplicity that the magnitude of $2 U$ is greater than the magnitude of $\omega R_{0}$.

Between the top and the bottom the pressure difference is

$$
p_{T}-p_{B}=-4 \rho U \omega R_{0}
$$

And the pressure gradient across the cylinder is

$$
\frac{p_{T}-p_{B}}{2 R_{0}}=-2 \rho U \omega
$$

Pressure is lower on top of the cylinder; the pressure force points up. Equation (5) gives the irrotational pressure force (Magnus force) on the cylinder along the vertical line passing through the cylinder's center. In general the speed and the frequency in (5) are independent variables (for rolling motion they are related).

\section{New Foundation}

Begin again with a steady frictionless flow, from left to right, past a cylinder that is not rotating. Far from the cylinder the velocity of the fluid is constant in magnitude, $U$, and uniform in direction, but this time no irrotational assumption is made. Normal to the mean flow and at the top of the cylinder the $z$-axis points up. In this two-dimensional problem the flow around the cylinder cross-section is in the $x$-z plane, and the long axis of the cylinder is in the $y$-direction.

Bernoulli's equation along any streamline going over the top of the cylinder is

$$
p=\text { const }-\frac{1}{2} \rho V^{2}
$$

where $p$ is the pressure, $V$ is the speed of the flow and $\rho$ is the fluid density, taken constant. For ease of calculation the constant in (6) is assumed the same for all streamlines.

Fluid following a curving path anywhere above the top of the cylinder experiences an upward centrifugal force which attempts to tear the fluid away from the solid and away from itself. When the flow is steady, as often observed at low speeds, there must be an equal but opposite force to balance the centrifugal force everywhere. It can only be a pressure gradient in this situation. Therefore the force balance is 


$$
\frac{\mathrm{d} p}{\mathrm{~d} z}=\frac{\rho V^{2}}{R}
$$

In (7) $R$ is the radius of curvature of the streamlines, which is the same as the radius of the cylinder at the very top of the cylinder. Adopting the convention that $R$ is positive, then the RHS of (7) is positive. Thus the left hand side (LHS) of (7) shows that the pressure must increase with increasing height over the cylinder, or that the perturbed pressure (the relatively low pressure at the cylinder's top) decreases upward.

Now, between (6) and (7) the pressure can be eliminated quickly, starting by taking the $z$ derivative of both sides of (6), substituting the pressure gradient from it into the pressure gradient in (7), to form a velocity equation for variations in the $z$-direction

$$
\frac{\mathrm{d} V}{\mathrm{~d} z}=-\frac{V}{R}
$$

Equation (8) is a first order ordinary differential equation with a non-constant coefficient, since $R=R(\mathrm{z})$ above the cylinder. Unlike either one of the two equations it came from Equation (8) is linear! However, in order to solve (8) completely information has to be provided about the radius of curvature of the streamlines.

\section{Discussion}

Observations (streak photographs [2]) qualitatively show that the radius of curvature of the streamlines above the top of a cylinder increases with increasing distance away from the cylinder until, only a few radii away, there is no longer any curvature left (the streamlines are straight). It is not obvious from the available data what the exact rate of increase is, although one might be able to say that the increase is faster than linear. Also a principle of physics does not occur at this time that could help determine the explicit rate of increase algebraically. Whatever the "true" vertical structure of the radius of curvature of the streamlines turns out to be, Equation (8) can be solved, if not analytically, then numerically, since only a first order ODE needs to be dealt with.

In order to make progress now, one of the simplest nonlinear functional forms of the increase of the radius of curvature with height above the cylinder, which makes the following calculations tractable, is selected for study

$$
R=R_{0}\left(1+\frac{z}{R_{0}}\right)^{3}
$$

In (9) $R_{0}$ is the radius of curvature of the cylinder. Putting (9) into the right hand side (RHS) of (8), using separation of variables, integrating both sides independently, and then raising both sides to the power of the exponential, the complete solution is

$$
V=V_{0}\left(\mathrm{e}^{f(z)}-1\right)+U
$$

where the constant to be determined shortly is $V_{0}$. The exponent in (10), using (9), is

$$
f(z)=\frac{1}{2}\left[\frac{1}{\left(1+\frac{z}{R_{0}}\right)}\right]^{2}
$$

More generally the exponent in Equation (10) can be expressed as

$$
f(z)=-\int_{0}^{z} \frac{d z^{\prime}}{R\left(z^{\prime}\right)}
$$

From which it can be seen that the solution to (10) is not sensitive to the particular path $R(z)$ takes between $R_{0}$ and $\infty$ because of the integration involved in the RHS of (12).

\section{Magnus Force}

To compute the Magnus force the constant in (10) needs to be determined. This can be done by conserving mass 
between two vertical cross-sections: one is directly over the top of the cylinder and the other is any vertical line away from the influence of the cylinder on the flow, starting at the horizontal line that passes through the middle of the cylinder and continuing to infinity. Having done that, the result is very nearly given by

$$
V_{0} \approx 2 U
$$

By the approximately equals sign in (13) is meant that only the first three terms in the expansion of the exponential term in (10), with (11) included, were kept in the calculation of conserving mass.

Inserting (13) into (10) and evaluating it at $z=0$ to give the maximum horizontal speed at the top of the cylinder results in

$$
V=2.3 U
$$

Which is $15 \%$ higher than the irrotational value $(V=2 U)$. A question that naturally comes up is: if a different function than (9) had been chosen to represent the radius curvature of the streamlines, hopefully in better agreement with observations, would the accordingly modified Equation (14) have come closer (or not) to equaling the irrotational result? Future work may provide the answer.

With (14) as is, and the cylinder now rotating, the Magnus force will be 15\% larger than obtained in (5).

Finally, it is known that the fall-off rate for velocity with distance away from the cylinder is as the inverse square of the distance in the irrotational solution. Equation (14) implies, however, that in model two the fall-off rate is faster than that in order to conserve mass.

\section{Conclusion}

Some of the confusion in the literature concerning the properties of the Magnus effect on rotating solid cylinders translating through a fluid may stem from the apparent lack of a published formula for the force. Two algebraic formulas are constructed for the Magnus force which agree in direction but differ in magnitude by $15 \%$. The first model, based on an existing irrotational solution of the problem, has the smaller force. In the second model irrotationality is not assumed but instead a new method is given involving the force balance between a pressure gradient and the centrifugal force on flow along curving streamlines above the cylinder's top. Observations are needed for comparison with the two different results. Basically the formula consists of the following factors multiplied together: constant, fluid density, translation speed, and rotation frequency. For the classical fluid model constant $=2.0$; for the new model constant $=2.3$.

\section{References}

[1] Schlichting, H. (1968) Boundary-Layer Theory. Mc Graw-Hill, New York, 364.

[2] Batchelor, G. K. (1967) An Introduction to Fluid Dynamics. Cambridge University Press, Cambridge, UK, plate 1.

[3] Tritton, D.J. (1988) Physical Fluid Dynamics. Oxford Science Publications, New York, 159-161.

[4] Faber, T.E. (1995) Fluid Dynamics for Physicists. Cambridge University Press, Cambridge, UK, 281. http://dx.doi.org/10.1017/cbo9780511806735

[5] Cullerne, J. (2009) Dictionary of Physics. Penguin, New York.

[6] Oxford English Dictionary (2007). Oxford, UK.

[7] Kenyon, K.E. (2013) Flow past a Cylinder. Journal of Scientific Theory and Methods, 2013, 211-222.

[8] Kundu, P.K. (1990) Fluid Mechanics. Academic Press, San Diego, 155. 